\title{
Systematic determination of the mosaic structure of bacterial genomes: species backbone versus strain-specific loops H Chiapello*1, I Bourgait ${ }^{1}$, F Sourivong ${ }^{1}$, G Heuclin ${ }^{1}$, A Gendrault- Jacquemard $^{1}$, M-A Petit ${ }^{2}$ and M El Karoui ${ }^{2}$
}

Address: ${ }^{1}$ Mathématique, Informatique \& Génome, INRA Domaine de Vilvert, 78352 Jouy-en-Josas cedex, France and ${ }^{2}$ Unité de Recherches Laitières et Génétique Appliquée, INRA Domaine de Vilvert, 78352 Jouy-en-Josas cedex, France

Email: H Chiapello* - helene.chiapello@jouy.inra.fr; I Bourgait - isabelle.bourgait@jouy.inra.fr; F Sourivong - fsourivong@wanadoo.fr; G Heuclin - g.heuclin@tele2.fr; A Gendrault-Jacquemard - annie.jacquemard@jouy.inra.fr; M-A Petit - marie-agnes.petit@jouy.inra.fr; M El Karoui - meriem.el-karoui@jouy.inra.fr

* Corresponding author

Published: 12 July 2005

BMC Bioinformatics 2005, 6:17| doi:|0.1|86/|47|-2105-6-17|
Received: 05 April 2005

Accepted: 12 July 2005

This article is available from: http://www.biomedcentral.com/I47I-2105/6/17I

(c) 2005 Chiapello et al; licensee BioMed Central Ltd.

This is an Open Access article distributed under the terms of the Creative Commons Attribution License (http://creativecommons.org/licenses/by/2.0), which permits unrestricted use, distribution, and reproduction in any medium, provided the original work is properly cited.

\begin{abstract}
Background: Public databases now contain multitude of complete bacterial genomes, including several genomes of the same species. The available data offers new opportunities to address questions about bacterial genome evolution, a task that requires reliable fine comparison data of closely related genomes. Recent analyses have shown, using pairwise whole genome alignments, that it is possible to segment bacterial genomes into a common conserved backbone and strainspecific sequences called loops.
\end{abstract}

Results: Here, we generalize this approach and propose a strategy that allows systematic and nonbiased genome segmentation based on multiple genome alignments. Segmentation analyses, as applied to 13 different bacterial species, confirmed the feasibility of our approach to discern the 'mosaic' organization of bacterial genomes. Segmentation results are available through a Web interface permitting functional analysis, extraction and visualization of the backbone/loops structure of documented genomes. To illustrate the potential of this approach, we performed a precise analysis of the mosaic organization of three $E$. coli strains and functional characterization of the loops.

Conclusion: The segmentation results including the backbone/loops structure of 13 bacterial species genomes are new and available for use by the scientific community at the URL: http:// genome.jouy.inra.fr/mosaic.

\section{Background}

Systematic genome comparisons play an increasingly important role in genome analysis and annotation. There are mainly two kinds of approaches used for whole genome comparisons: whole proteome comparison studies and whole genomic sequence alignment studies. Both approaches are powerful tools to study genome organiza- tion and evolution rules with different time scale considerations. These approaches have been employed with success in a recent study comparing the genome of yeast $S$. cerevisiae to three related yeast species genomes $[1,2]$. Genomewide comparative analysis of the yeast chromosomes has considerably improved gene annotation and has permitted the prediction of new motifs conserved in 
intergenic regions that act potentially as regulatory elements of gene expression [1].

Whole genome-alignments tools have shown important developments in the last years. It is now possible to align rapidly two or more long genomic DNA sequences with several tools like MultiPipMaker [3], Vista [4], Mummer [5-7] and MGA [8]. Some of them include graphical interfaces to display and browse genome alignments $[3,4,7]$. Other resources provide precomputed alignments for genome of related species, such as EnteriX or Colibase for enterobacteria $[9,10]$.

Here we focus on whole genomic sequence alignments in the particular case of strains of single bacterial species. Since the publication of a second strain of Helicobacter pylori in 1999 [11], sequence data on closely related bacterial genomes has rapidly accumulated in public databases. The availability of complete genome sequences for multiple strains of numerous species opens up new perspectives for studying short term evolutionary processes. For example pairwise alignment of the complete genomes of the enterohemorrhagic Escherichia coli 0157:H7 strains (Sakaï or EDL 933) with the E. coli K-12 laboratory strain, allowed the definition of a $4.1 \mathrm{Mb}$ sequence that was highly conserved between the two strains $[12,13]$. It was proposed that this common sequence corresponds to the conserved backbone of the E. coli chromosome, which is interrupted by numerous DNA segments called strain-specific loops, distributed throughout the backbone [12].

Examination of "mosaic" structures of backbones and loops offers a potential approach to trace the dynamics of genome evolution at the bacterial species level. The backbone, conserved in all aligned genomes of the species, probably corresponds in large part to the common ancestral strain and is the part of the genome under vertical selective pressure. As such, the backbone is also likely to be the most adapted part of the genome, which could be relevant when studying essential functional elements of the cell (such as genes, motifs or signals). Loops differ among strains. Some may correspond to mobile elements, like prophage [14] and insertion sequences [15], and may be associated with strain-specific pathogenicity. However little is known about functional elements associated with small loops.

Up to now, no systematic strategy for backbone/loop segmentation has been proposed for closely related bacterial genomes. The existing studies are either limited to pairwise comparisons or choose a reference genome which is compared to several related genomes. Precomputed alignments are often limited to a subgroup of species and use different softwares and parameters, making results generally non-reproducible or non-comparable.
In this paper we address the problem of defining a strategy to obtain a backbone/loop segmentation of bacterial genomes at the intraspecies level. This approach is based on two recent genome aligners: Mummer3 [7] and MGA [8]. Using a validated benchmark dataset, we developed a simple treatment of alignment results which permits a robust definition of the mosaic structure. Our approach does not take any genome as a reference and has no restriction for the number of genomes to align. We used our method to define this segmentation for 13 bacterial species. Validated backbone/loop segmentation results are stored in the MOSAIC database and are freely accessible through a user friendly Web interface. The backbone/ loop segmentation determined using three E. coli genomes illustrates important properties of this structure, and indicates that intraspecies segmentation is a useful mean of enhancing bacterial genome annotation.

\section{Results}

The global strategy of genome segmentation and database integration used in this study is outlined Figure 1. A reference set, consisting in a manually verified genome alignment, was used to set appropriate segmentation parameters. Using this strategy, alignments and segmentations were performed systematically for 13 bacterial species, for which at least two genomes have been sequenced. Loop and backbone coordinates were then integrated in the MOSAIC database together with NCBI genome annotations.

\section{Validation of segmentation parameters}

The loop coordinates of the E. coli K-12 and O157:H7 Sakai genomes validated by Hayashi et al. [16] (see Methods) were used as a basis to define an alignment strategy and to develop a treatment of alignment results adapted to bacterial backbone/loop segmentations. These strains are known to belong to distantly related $E$. coli lineages [17] and their genomes are more distantly related between each other compared with genomes within other species [18]. Parameters allowing a pertinent alignment of such different genomes were therefore expected to produce reliable results for more closely related strains. The K-12/Sakai comparisons were performed using different parameters of the MGA software, and those leading to the best results, as compared to coordinates obtained by Hayashi $e t$ al., were chosen. This set was used to produce alignments for all species so that results may be compared.

MGA software provides three types of results: matches (anchored MEMs of a minimal given length), aligned gaps (segments between anchored MEMs, shorter than a userdefined size and aligned with ClustalW) and unaligned gaps. Matches were computed using an iterative process on MEM size: MEM of at least $50 \mathrm{bp}$ were used for the first MGA step and MEM of at least 20 bp were computed in 


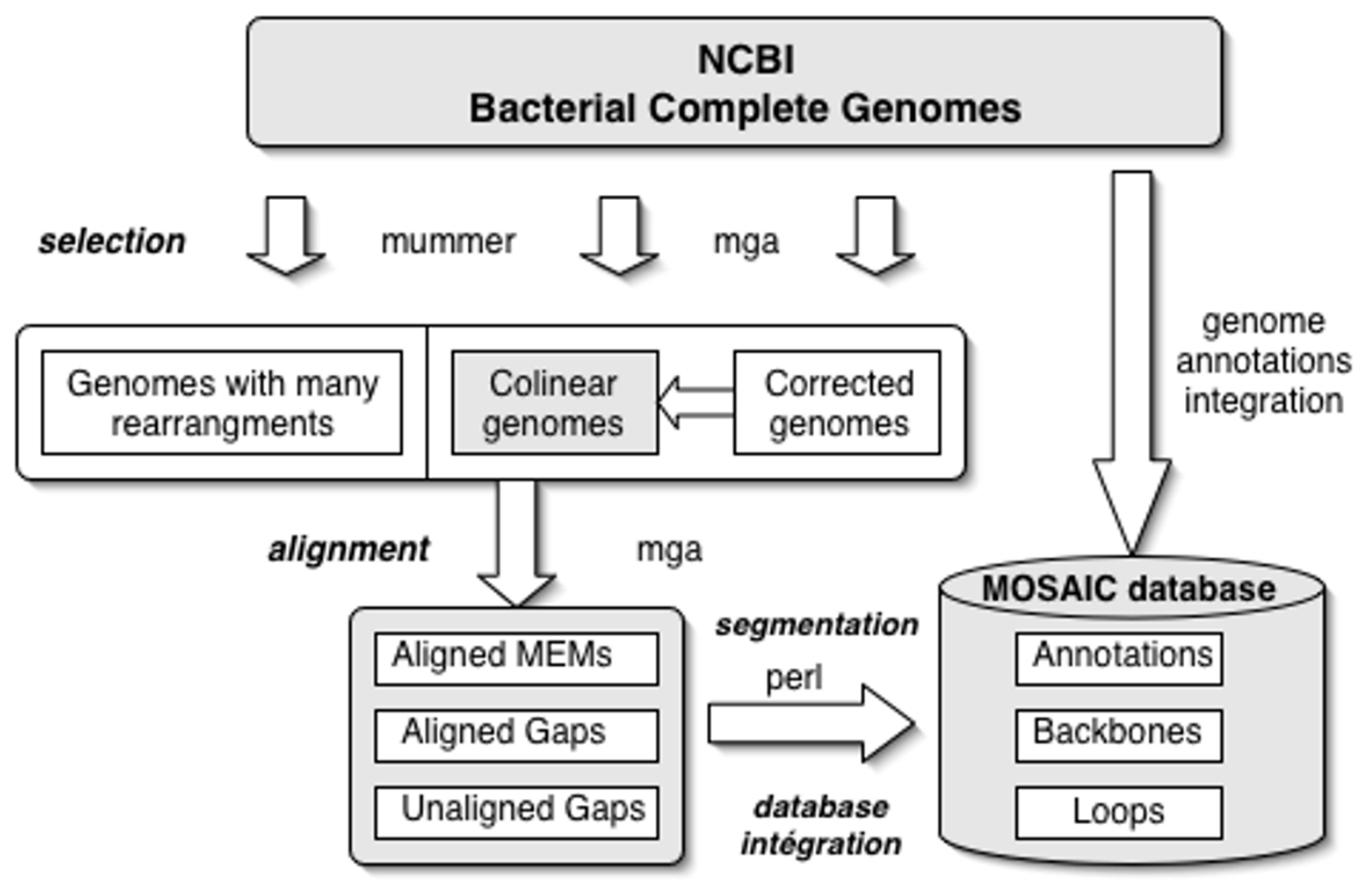

Figure I

Flow diagram of bacterial genomes segmentation in MOSAIC. The bacterial genomes segmentation includes four main steps in MOSAIC: NCBI bacterial genomes selection using Mummer and MGA, processing of genome alignments using MGA, backbone/loops segmentation and database integration using Perl scripts.

the second recursive step. These two kinds of MEM were included into the backbone of the segmented genomes. The gaps were then treated as follows : gaps longer than 3000 bp (unaligned MGA gaps) were considered as loops, and gaps shorter than 3000 bp were aligned with ClustalW. Aligned gaps with more than $76 \%$ identity were considered as backbone, others, as loops. Minimal size of loops and backbone segments was set to 20 nucleotides each. This strategy generated a backbone/loop profile of the K-12/Sakai alignment that differed by around $2400 \mathrm{nt}$ $(0,1 \%)$ from that validated by Hayashi et al. [16]

\section{Genome selection for the backbone/loop segmentation}

In order to select a subset of genomes for which backbone/loop segmentation makes sense, an analysis using the Mummer package was performed (see Methods). Three categories of results we obtained. The first category includes 33 genomes for which MGA alignments and backbone/loop segmentations are feasible, as they have not been submitted to numerous and important rear- rangements. The second category includes genomes that can be aligned after minor correction of their sequences (Reverse complement and Translation operators, see Material \& Methods section). This second category concerns 5 genomes ( 4 species). The last category corresponds to 17 genomes belonging to 8 species: Neisseria meningitis, Prochlorococcus marinus, Salmonella enterica, Shigella flexneri, Streptococcus pyogenes, Tropheryma whipplei, Xylella fastidiosa and Yersinia pestis. These genomes were excluded because Mummerplot results revealed rearrangements covering a large part of the genome.

\section{Genome alignments and backbone/loop segmentation}

Twenty four genome alignments were generated and treated for backbone/loop segmentation using MGA and our defined set of parameters. These included two quadruple alignments (E. coli, C. pneumoniae), four triple alignments (C. pneumoniae, E. coli, S. aureus, S. pyogenes) and eigthteen pairwise alignments. For one species, Buchnera aphidicola, segmentation results were not exploited due to 
Table I: Segmentation results obtained from MGA alignments and included in the MOSAIC database. For each segmentation result, the first column describes the species and genomes used for segmentation analyses; the number of compared strains is indicated between parentheses. Total loop sizes and loop number of each genome are entered in the same order as strain names, and separated by ' + '. Coverage corresponds to the ratio between backbone size and total genome size of a strain; here the mean value for all compared strains is given in percents.

\begin{tabular}{|c|c|c|c|}
\hline Compared genomes (numbers of strains) & Backbone size (Mb) & Cumulative loop size (kb) [Loop number] & Coverage (mean) \\
\hline \multicolumn{4}{|l|}{ Agrobacterium tumefaciens } \\
\hline C58 Cereon circ X C58 Univ. Wash circ (2) & 2.09 & $751[24]+751[25]$ & $74 \%$ \\
\hline C58 Cereon lin RC X C58 Univ. Wash lin (2) & 1.82 & $252[13]+253[13]$ & $88 \%$ \\
\hline \multicolumn{4}{|l|}{ Bacillus anthracis } \\
\hline Ames $X$ Ames 'Ancestor' (2) & 3.93 & $528[26]+528[24]$ & $90 \%$ \\
\hline \multicolumn{4}{|l|}{ Bacillus cereus } \\
\hline ATCCI4579 X ATCCI0987 (2) & 4.02 & $1390[2878]+1203[2872]$ & $76 \%$ \\
\hline \multicolumn{4}{|l|}{ Chlamydophila pneumoniae } \\
\hline AR39 RC+TR X CWL029 XJ138X TWI83 (4) & 1.22 & $10[13]+10[13]+7[11]+6[12]$ & $99 \%$ \\
\hline CWL029 XJI38 X TWI83 (3) & 1.21 & $15[14]+11[12]+10[13]$ & $99 \%$ \\
\hline CWL029 X JI38 (2) & 1.21 & $21[15]+17[14]$ & $99 \%$ \\
\hline JI38 X TWI83 (2) & 1.22 & $9[9]+8[10]$ & $99 \%$ \\
\hline CWL029 X TWI83 (2) & 1.22 & $13[6]+9[6]$ & $99 \%$ \\
\hline AR39 RC+TR X CWLO29 (2) & 1.22 & $8[7]+8[7]$ & $99 \%$ \\
\hline \multicolumn{4}{|l|}{ Escherichia coli } \\
\hline K-12 X Sakai X EDL933 X CFT073 (4) & 3.52 & $1119[848]+1978[830]+2008[830]+|7| 1[8 \mid 1]$ & $68 \%$ \\
\hline K-12 X Sakai X CFT073 (3) & 3.73 & $904[827]+1763[795]+1496[770]$ & $73 \%$ \\
\hline \multicolumn{4}{|l|}{ Helicobacter pylori } \\
\hline $26695 \times 199(2)$ & 1.24 & $428[957]+403[967]$ & $75 \%$ \\
\hline \multicolumn{4}{|l|}{ Listeria monocytogenes } \\
\hline EGD $\times 4 b$ F2365 (2) & 2.67 & $270[644]+230[638]$ & $92 \%$ \\
\hline \multicolumn{4}{|l|}{ Mycobacterium tuberculosis } \\
\hline CDCI55I X H37Rv (2) & 4.19 & $217[164]+225[162]$ & $95 \%$ \\
\hline \multicolumn{4}{|l|}{ Staphylococcus aureus } \\
\hline MW2 X MU50 X N315 (3) & 2.59 & $226[388]+283[382]+220[388]$ & $92 \%$ \\
\hline \multicolumn{4}{|l|}{ Streptococcus agalactiae } \\
\hline 2603V/R X NEM3I6 (2) & 1.88 & $276[135]+327[132]$ & $86 \%$ \\
\hline \multicolumn{4}{|l|}{ Streptococcus pneumoniae } \\
\hline R6 X TIGR4 (2) & 1.91 & $128[282]+250[294]$ & $91 \%$ \\
\hline \multicolumn{4}{|l|}{ Streptococcus pyogenes } \\
\hline MIGAS X MGAS3I5 X MGAS8232 (3) & 1.62 & $235[275]+283[282]+277[282]$ & $86 \%$ \\
\hline MIGAS X MGAS3I5 (2) & 1.64 & $210[191]+258[192]$ & $88 \%$ \\
\hline MIGAS X MGAS8232 (2) & 1.65 & $206[225]+249[231]$ & $88 \%$ \\
\hline \multicolumn{4}{|l|}{ Vibrio vulnificus } \\
\hline YJ016 K2 X CMCP6 K2 TR (2) & 1.63 & $222[198]+210[199]$ & $89 \%$ \\
\hline YJ016 KI RC X CMCP6 KI TR (2) & 2.73 & $628[340]+555[338]$ & $82 \%$ \\
\hline
\end{tabular}

too low coverage (this value estimates the percentage of total genome length included in the backbone, in this case $40 \%$, see Discussion).

Validated segmentation results including backbone size, loop size, loop number and genome coverage are described in Table 1 . The coverage ranged from $68 \%$ for E. coli quadruple alignment to $99 \%$ for C. pneumoniae strains. Species comparisons giving high coverage values may be a consequence of to the choice of closely related strains for sequencing, but may also indicate that overall horizontal transfer is less important in some species than in others.
The number of loops in a segmented genome appeared to be also highly variable among bacterial species, ranging from 6 (Chlamydophila pneumoniae strain CWL29 compared to strain TW183) to 2878 (Bacillus cereus, strain ATCC14579 compared to ARCC10987). Results of table 1 revealed two extremes situations. Some species have few very long loops, as Agrobacterium tumefaciens (24/25 loops for the circular chromosome, mean length of loops around 28 kilobases). Others (Bacillus cereus) contain a large number of short loops (mean length around 400 bases). These differences will need to be further examined in details, in relation with genome annotations. 


\section{Database integration and web interface}

Alignments were integrated into the MOSAIC database and are accessible through the Web interface. Access to the mosaic structure of genomes is made by species selection or gene name selection. For each segmented genome, a local view of the physical map of the segmented genome is available, using MuGeN software [19] (see Figure 2). This graphical visualization of loop and backbone structures is associated with Genbank/NCBI genome annotations. In addition, an overall graphical view of backbone and loops structure is presented using EMBOSS cirdna program. Finally, lists of loop and backbone segments can also be inspected and downloaded according to different criteria like size, genome position or functional characterization.

\section{In depth analysis of the backbonelloop structure of three E. coli genomes}

A more precise analysis of the segmentation results from the comparison of E. coli strains K-12 [20], O157:H7 Sakai [12] (named Sakai below) and CFT073 [21] (named CFT below) was performed. A $3.73 \mathrm{Mb}$ length backbone (exhibiting more than $97 \%$ identity between the three strains) and three sets of strain-specific loops (of very different total length) were identified. The K-12 genome included $827 \mathrm{~K}-12$ loops (total length $0.9 \mathrm{Mb}, 20 \%$ of the K-12 genome), the Sakai genome, 795 Sakai loops (total length $1.8 \mathrm{Mb}, 33 \%$ of the Sakai genome) and the CFT genome, $770 \mathrm{CFT}$ loops (total length $1.5 \mathrm{Mb}, 29 \%$ of the CFT genome). The differences in total loop sizes are in keeping with the different total genome size of the three strains (K-12: 4.6 Mb ; Sakai: 5.5 Mb ; CFT: 5.2 Mb).

\section{A large proportion of short loops in the E. coli genome} Basic statistics concerning the size distribution of the three loop sets are described in Table 2 and Figure 3. Results of Table 2 show a remarkable number of short loops for the three species: three quarters of the K-12 loops are shorter than 486 nucleotides (respectively 863 and 314 for Sakai and CFT). The histogram of loop size distribution for the three E. coli strains (Figure 3) reveals that the loop population is heterogeneous. Interestingly it appears that the loop populations exhibit roughly the same profile in the three strains, which may comprise three sub-populations: numerous very short loops (length around $100 \mathrm{bp}$ ), medium-size loops (length around 1-2 kb) and a few very long loops (length > $10 \mathrm{~kb}$ ). This may reflect a wide diversity of functional properties conferred by loops: the longer loops probably encode several genes (and correspond for example to bacteriophage or pathogenicity islands). The shorter ones might have regulatory roles or affect gene expression.

\section{Functional elements associated with backbone and loops}

The distribution of functional elements in the backbone/ loop structure was analyzed. Functions identified by classical annotations of bacterial genomes i.e. genes, tRNA, rRNA, phages and Insertion Sequences (IS) were first considered. As expected tRNA and rRNA were mainly present in the backbone. One exception concerns a rather large proportion of tRNA present in the Sakai loops (27\%) compared to K-12 (9\%) and CFT (13\%) loops. The Sakai strain contains 18 specific tRNAs not present in K-12 [12], Hayashi et al. observed that these tRNAs recognize codons which are used with an increased frequency in Sakai loops. Not surprisingly, we observed that phages and IS are quasi-totally included in loops (>98\%). The ten longest loops of K-12 correspond systematically to known phages, or phage remnants of the E. coli genome.

\section{One hundred E. coli K-I 2 loops are associated with BIME}

To refine functional categorization of smaller loops we examined the correspondence between loops and Bacterial Interpersed Mosaic Element (BIME). BIME are short palindromic repetitive DNA elements found in the genomes of E. coli and other enterobacteria [22], and are present exclusively in intergenic regions. BIME are composed of three types of palindromic units (Y, Z1 and Z2). Three sub-families of BIME have been described: BIME-1, which are composed of one $\mathrm{Y}$ and one Z1; BIME-2 ("composite" BIME) which contain two to twelve Y and Z2; and a third category ("atypical BIME"), which refers to all other palindromic units associations. BIME sizes range from $140 \mathrm{bp}$ for BIME-1 to several kb for BIME-2 or atypical BIME. BIME are reported to have several functions: mRNA stabilization, transcription termination, translational control and genomic rearrangements [22]. Using the MOSAIC database we identified 100 loops associated with BIME. BIME coordinates were obtained from the "short repeated palindromes in enterobacteria" Web site [23]. They are distributed as follows: in 31 cases, a BIME was present within the loop. In 29 cases, the BIME covered the entire loop region and extended into flanking backbone sequences. In 40 cases, the BIME accounted for more than $50 \%$ of the loop length and extended over to one side of the backbone. Results concerning BIME distribution in backbone and loops on the K-12 genome (Table 3 ) clearly indicate that loops are enriched in BIME elements: in particular 2/3 of the DNA regions associated to BIME are located on the loops. This tendency is particularly striking for BIME-1, for which $71 \%$ of the cumulated length is associated with loops. Interestingly, BIME-2 are quasi equally distributed between backbone and loops. This result is a generalization to all K-12 BIMEs of a result observed in a previous work [24]. PCR analysis of 3 BIME1 and 3 BIME-2 loci in $51 \mathrm{E}$. coli and Shigella isolates showed that BIME-1 are either present or absent among isolates whereas BIME-2 are generally present in the same 

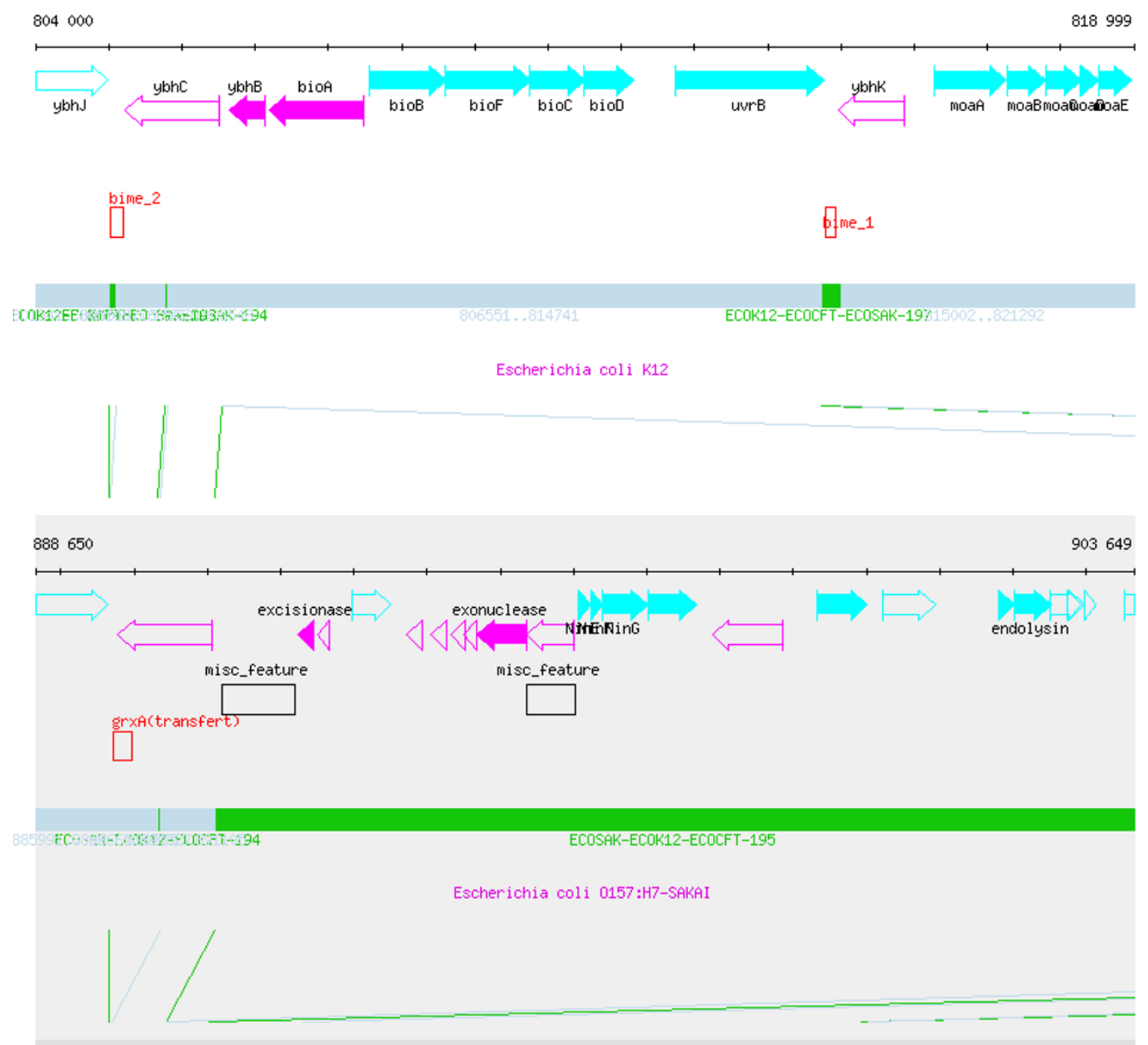

827016

842015
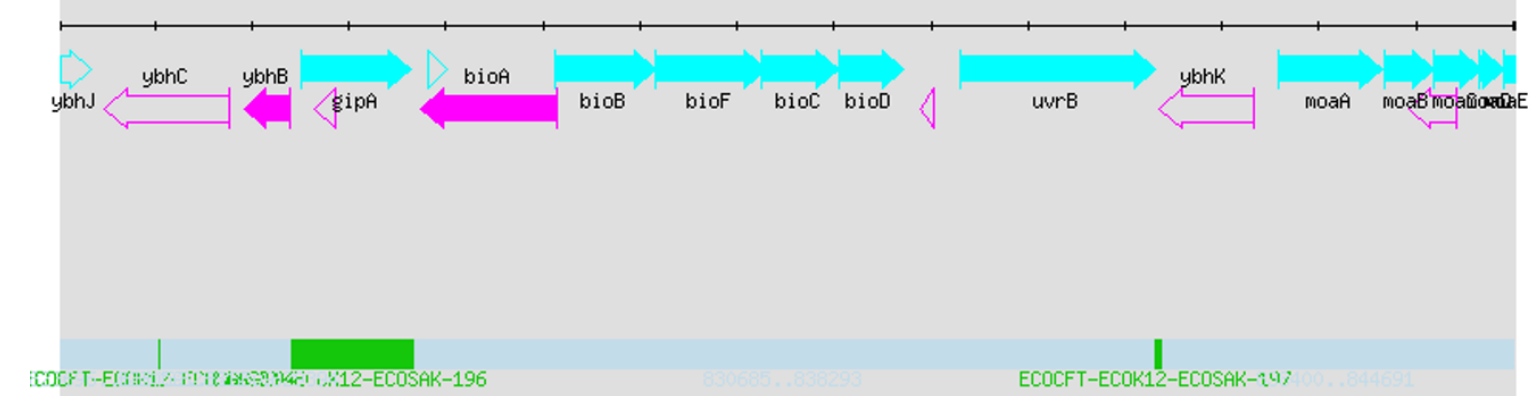

ECOCFT-ECOK12-ECOSAK-१७;

Escherichia coli CFT073

\section{Figure 2}

Graphical visualization of the backbone/loop structure available through the Web interface of Mosaic. 'Physical map' mode of MOSAIC corresponding to the graphical visualization of a $15 \mathrm{~kb}$ portion of the E. coli K-I2, OI57:H7 Sakai and CFT073 segmented genomes (data correspond to the comparison of three E. coli strains described in results). Genbank annotations are indicated with coloured arrows. Supplementary annotations are indicated as red boxes. Backbone is indicated in grey whereas loops appear in green. 
Table 2: Size distribution of loops (in bp) obtained from segmentation of the E. coli genomes K-12, OI57:H7 Sakai (SAK) and CFT073 (CFT). Minimal size (Min), Mean size, Maximal size (Max), First Quartile (Ist Qu.), Median size, and Third Quartile (3rd Qu.) are shown.

\begin{tabular}{llll}
\hline & K-I2 loops & SAK loops & CFT loops \\
\hline Min & 20 & 20 & 20 \\
Mean & 1093 & 2217 & 1942 \\
Max & 40120 & 96682 & 150690 \\
Ist Qu. & 34 & 32.5 & 31 \\
Median & 113 & 109 & 77 \\
3rd Qu. & 486 & 863 & 314 \\
\hline
\end{tabular}
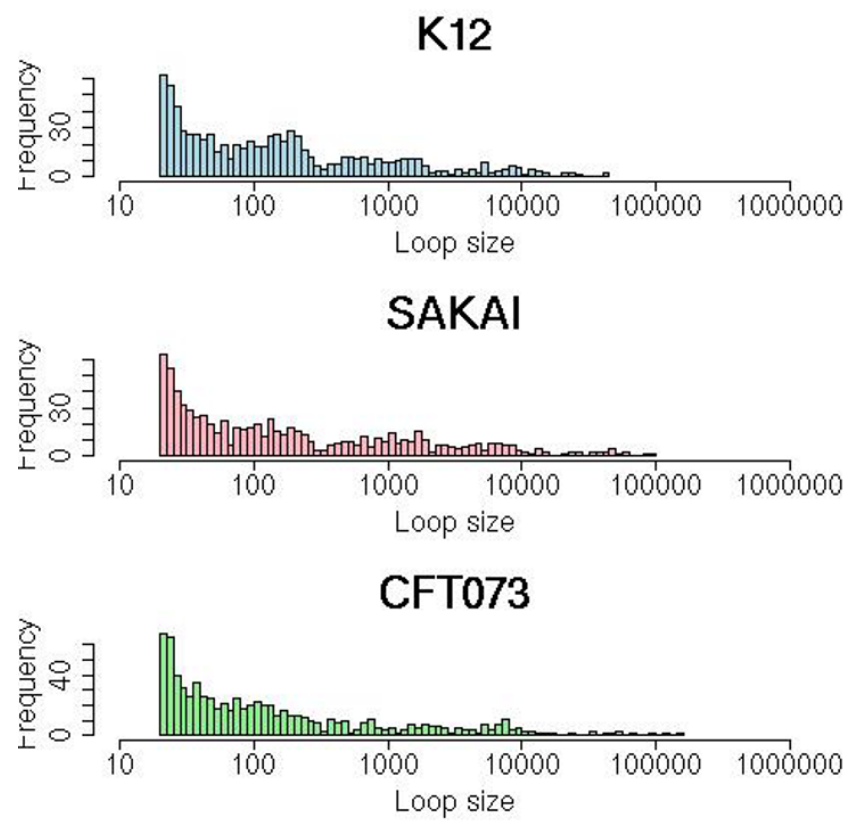

Figure 3

Distribution of the loop sizes of three E. coli genomes (K-1 2, SAKAI and CFT073). Loop sizes range from 20 bp to 40 I 20 to I5I $690 \mathrm{bp}$. Log 10 scale is used on the $x$-axis.

set of isolates but exhibit a high level of length polymorphism [24]. Figure 2 illustrates two examples of loops associated with BIME-1 and BIME-2, as visible with MuGeN through the MOSAIC Web interface. This association of loops with BIME is a first clue in characterizing the functionality of short loops in E. coli strains.
Table 3: Distribution of BIME (in percent of length) in backbone and loops regions of the $E$. coli K- 12 genome, as determined from the triple K-I 2, Sakai and CFT073 alignment.

\begin{tabular}{lll}
\hline & K-I2 & \\
\hline & Backbone & Loops \\
\hline BIME & $38 \%$ & $62 \%$ \\
BIME I & $29 \%$ & $71 \%$ \\
BIME 2 & $47 \%$ & $53 \%$ \\
atypical BIME & $37 \%$ & $63 \%$ \\
\hline
\end{tabular}

\section{Discussion}

Backbone/loop segmentation as a step towers analysis of genome evolution

Studying backbone and loops of bacterial genomes is an efficient way to distinguish the two major modes of evolution acting on bacterial genomes. The backbone may be considered as the part of the genome susceptible to vertical long-term evolution. Backbones are very similar for closely related strains and variability comes mainly from punctual mutations or insertions/deletions of oligonucleotides. The loop population (defined in MOSAIC as variable regions of $21 \mathrm{bp}$ or more) is more heterogeneous : the number of loops and the average loops length varies greatly from one species to another (Table 1). Loops can be viewed as elements issued from short-term evolution processes. One such process is horizontal transfer. For example acquisition/loss of distinct prophage sets seems to be a rapid process, which can be observed between closely related strains [25]. Significantly, for some genomes, phages are the major contributors to loop length [14]. Eleven loops of E. coli K-12 are associated with phages and constitute $24 \%$ of the total E. coli K-12 loop length. A contrasting example is found in $H$. pylori: this species does not contain prophage, although it contains large loops that may be associated with pathogenicity islands [11]. Our results indicate that medium-size loops (scale of the gene size) are constituted, at least in part, from known variable elements of bacterial genomes like Insertion Sequences. The relatively large number of short loops found in some species (E. coli, B. cereus) is quite surprising. Such small loops may be due to replication errors ('copy-choice' of DNA polymerase, slippage mechanism), which can generate small insertions or deletions [26] or may correspond to highly polymorphic regions. As opposed to large or medium size loops it is likely that these shorter loops arose from non-horizontal transfer events. 


\section{Consequences of segmentation from multiple alignments} Alignments including more than two genomes generally yield a more robust but smaller backbone than pairwise alignments. This is due to the fact that a larger set of genome variations is taken into account. In the future, about ten or more genomes will be available for some species. One possible consequence is that the backbone length will shrink steadily with new strain genomes. In that case, the backbone may rather be redefined as, for example, the subset of chromosomal regions present in at least half of the strains. Alternatively, the backbone size may decrease but rapidly reach a minimal size, which will be stable even when new strain genomes will be added for alignment.

As a consequence of multiple comparisons, loop populations are greater and more heterogeneous. They include for example elements present in only one genome (which may correspond to acquisition of a very specific characteristic by one strain), elements present in a subset of strains or elements present in all genomes but one (which may correspond to a deletion in one strain). It will be important to systematically classify loops obtained from multiple comparisons in order to facilitate their identification through the MOSAIC interface.

To estimate the importance of loops corresponding to DNA present in the common ancestor but lost in one of the compared strains a preliminary study was performed: all sequences present in the K-12 loops (from the triple alignment) were blasted against the Salmonella typhimurium genome (considered as the outgroup), and matching sequences present in the same genome environment were considered as "ancestral loops". Ten loops, corresponding to a total length of $3658 \mathrm{bp}$, matched this criterium. This suggests that only a minor subset of the loops correspond to deletions that occurred in either $E$. coli Sakai or CFT genomes.

\section{Backbone/loops segmentation for divergent or rearranged genomes}

Some genomes of species like Buchnera aphidicola were too divergent to be segmented with our procedure. In fact, these genomes are clearly atypical in terms of evolutionary distance within a species: despite complete colinearity of their genomes, B. aphidicola Sg and Ap genomes display a high degree of divergence at the nucleotide level, making them as different as E. coli/S. typhimurium genomes [27]. Comparison of $\mathrm{Sg}$ and Ap genomes is thus almost the same situation as comparing different species, but would be possible by adapting the alignment parameters. This raises the question of bacterial species definition: the evolutionary distances within a species and between species are very heterogeneous. For example, it has recently been confirmed that Shigella is phylogenetically indistinguisha- ble from E. coli [28]. Our method will also be easily extended to bacterial species where numerous chromosomal rearrangements have occurred, using recently developed genome aligners such as MAUVE [29]. Intraspecies comparison of divergent and/or rearranged genomes will open the way to segmentation of genomes from different, but closely related species.

\section{A new category of genome annotation}

To our knowledge, this work is the first study allowing systematic mosaic genome segmentation of all available strains (ranging from two to four) in 13 bacterial species. Examination of the backbone of a bacterial species should greatly facilitate refinement of gene annotation and prediction of conserved sites with potential regulatory roles. Examination of the gene content in loops is important for identification of putative horizontally transferred genes. Genes adapted to specific ecological environments or involved in pathogenicity of a specific strain should also be found in the strain-specific loops. Indeed, the ASAP database (A Systematic Annotation Package for community analysis of genomes) [30] recently added the features type 'island' and 'conserved_segments' in order to provide lists of regions that are specific or common to the two $E$. coli K-12 and O157:H7 genomes.

\section{Conclusion}

Genome aligners were used to build a robust strategy for bacterial genome segmentation. Backbone/loops structures were systematically determined for 38 bacterial genomes. The MOSAIC resource makes it easy to visualize, annotate, and analyse loops and backbone segments of these genomes. First analyses reveal a surprising diversity in the number of loops from one species to another. In addition some species accumulate a large number of short loops, unsuspected previously.

\section{Methods}

\section{Species selection}

Complete bacterial genomes were downloaded from the NCBI microbial genome database: http:// www.ncbi.nlm.nih.gov/genomes/lproks.cgi?view=1, version of 06/24/2004. Twenty one species (55 genomes) for which genome sequences of at least two different strains are available were selected for analysis: Agrobacterium tumefaciens, Bacillus anthracis, Bacillus cereus, Buchnera aphidicola, Chlamydophila pneumoniae, Escherichia coli, Helicobacter pylori, Listeria monocytogenes, Mycobacterium tuberculosis, Neisseria meningitidis, Prochlorococcus marinus, Salmonella enterica, Shigella flexneri, Staphylococcus aureus, Streptococcus agalactiae, Streptococcus pneumoniae, Streptococcus pyogenes, Tropheryma whipplei, Vibrio vulnificus, Xylella fastidiosa, and Yersinia pestis [see Additional file 1]. 


\section{Segmentation strategy}

Backbone/loop segmentations were determined using a simple procedure based on Mummer3 [7] and MGA [8] results (see figure 1 ).

\section{Selection of genomes without rearrangement}

In the first step, the subset of genomes for which it is possible to define a reliable backbone was identified using mummer and mummerplot scripts of the Mummer3 package. First, all Maximal Exact Matches (MEM, not necessarily unique) of at least $20 \mathrm{bp}$ in both forward and reverse strands of the compared genomes were computed using the mummer program. Visualization of results between each pair of sequences was then performed using the mummerplot program. This graphical visualization was used to decide whether a common backbone could be defined for the considered genomes. In several cases, this step led us to adjust one of the genomes before the segmentation step. Two operators were defined: the reverse complement operator, named $\mathrm{RC}$, and the translation operator, named TRx, where $\mathrm{x}$ indicates that bases from position 1 to $\mathrm{x}$ were transferred to the end of the genome. This number $\mathrm{x}$ of bases shifted to the end of the genome was determined by the position of the first aligned MEM detected by MGA between the two genomes. These operators allowed us to assign the same strand and the same start position to all compared genomes. They were applied to a subset of genomes before alignment with MGA software. Genomes where rearrangements covering more than half of the total length were detected by mummerplot and excluded at this step. They can not be handled properly by MGA and would therefore lead to inaccurate segmentation.

\section{Backbone/loop segmentation}

The second step was to use the MGA software to perform whole genome alignments on the subset of selected genomes and to define backbone and loops. MGA is a powerful multiple genome aligner which presents two major advantages. First, it performs simultaneous multiple alignments based on MEM (Maximal Exact Matches present in all aligned genomes) selection, without considering any genome as the reference. Second, a consistent and robust backbone for the aligned genomes can be defined using its MEM anchoring algorithm followed by treatment of gaps (i.e. regions between the anchored MEM). Parameters used in MGA were adjusted by comparison with a manually curated reference set of loops of two E. coli strains: K-12 and O157:H7 Sakai [16]. After Mummer 1 alignment, backbone/loop junctions were extracted and systematically aligned using the fasta3 algorithm. Each alignment was checked by eye inspection and in many cases, the backbone sequence was extended by a few to several base pair [Pr. T. Hayashi, personal communication]. Further analysis using whole genome PCR scan- ning confirmed that the loops longer than $500 \mathrm{pb}$ are indeed variable elements [31]. A simple treatment of MGA alignment results was developed to define the boundaries of loops and to enhance their concordance with this manually determined pairwise reference dataset [see 'Results' section, 'Validation of segmentation parameters' subsection]

\section{Coverage calculation and database integration}

Results of MGA alignments were generated in XML format. Backbone/loop segmentations were processed with a Perl script using the SAX module for XML parsing. For each aligned genome, backbone and loop coordinates were computed and coverage (length of the backbone divided by total length of the genome) was calculated. Results were then integrated into the MOSAIC relational database. The database was implemented using the PostgreSQL relational database system. The MOSAIC relational model is generic and not dedicated to any alignment tool or genome species. The Web interface was also written in Perl language using standard modules for database access (DBI module for DataBase Interface) and dynamic pages (CGI module for Common Gateway Interface). Different graphical visualizations of the backbone/ loop structure were developed using the MuGeN software [19] and the cirdna program which is part of the EMBOSS package [32].

\section{Authors' contributions}

H. Chiapello performed segmentation results, conceived the MOSAIC application and drafted the manuscript. I. Bourgait, and A. Gendrault-Jacquemard participated in the database design and segmentation results integration. G. Heuclin performed loop analysis. F. Sourivong built the Web interface. M-A. Petit participated in data analysis and helped to draft the manuscript with M. El Karoui who supervised the study.

\section{Additional material}

\section{Additional File 1}

The 55 bacterial genomes for which at least two strains have been sequenced. For each species and each strain, NCBI accession number and genome length are indicated. The 'MGA aligt.' column indicates if the genomes have been included in an MGA alignment. Genomes corrections are indicated in the 'Correction column' as follows: '-', no correction, ' $R C$ ', Reverse Complement strand, and 'TR+x' means that segment in position 1 to $x$ of the genome has been shifted at the end of the genome. A brief comment is given for genomes excluded from MGA alignments. Click here for file

[http://www.biomedcentral.com/content/supplementary/14712105-6-171-S1.doc] 


\section{Acknowledgements}

We thank C. Hennequet-Antier for her assistance in using $R$ software and Dr A. Gruss for many helpful discussions. We are indebted to Professor T. Hayashi and Dr K. Kurokawa for sharing data before publication and helpful discussions. This work is supported in part by the "ACI IMPbio" grant from the French Ministry of Research.

\section{References}

I. Kellis M, Patterson N, Endrizzi M, Birren B, Lander ES: Sequencing and comparison of yeast species to identify genes and regulatory elements. Nature 2003, 423(6937):24I-54.

2. Kellis M, Patterson N, Birren B, Berger B, Lander ES: Methods in comparative genomics: genome correspondence, gene identification and regulatory motif discovery. J Comput Biol 2004, I I(2-3):319-355.

3. Schwartz S, Elnitski L, Li M, Weirauch M, Riemer C, Smit A, Green ED, Hardison RC, Miller W: MultiPipMaker and supporting tools: Alignments and analysis of multiple genomic DNA sequences. Nucleic Acids Res 2003, 3 I (1 3):35 I 8-24. 2003 Jul I

4. Frazer KA, Pachter L, Poliakov A, Rubin EM, Dubchak I: VISTA: computational tools for comparative genomics. Nucleic Acids Res 2004, 32(Web Server issue):W273-9. 2004 Jul I

5. Delcher AL, Kasif S, Fleischmann RD, Peterson J, White O, Salzberg SL: Alignment of whole genomes. Nucleic Acids Res 1999, 27(I I):2369-76

6. Delcher AL, Phillippy A, Carlton J, Salzberg SL: Fast algorithms for large-scale genome alignment and comparison. Nucleic Acids Res 2002, 30(I I):2478-83.

7. Kurtz S, Phillippy A, Delcher AL, Smoot M, Shumway M, Antonescu C, Salzberg SL: Versatile and open software for comparing large genomes. Genome Biol 2004, 5(2):RI2

8. Höhl M, Kurtz S, Ohlebusch E: Efficient multiple genome alignment. Bioinformatics 2002, I 8(SuppI I):S3 I 2-20.

9. Florea L, McClelland M, Riemer C, Schwartz S, Miller W: EnteriX 2003: Visualization tools for genome alignments of Enterobacteriaceae. Nucleic Acids Res 2003, 3 I(I3):3527-32. 2003 Jul I

10. Chaudhuri RR, Khan AM, Pallen MJ: ColiBASE: an online database for Escherichia coli, Shigella and Salmonella comparative genomics. Nucleic Acids Res 2004, 32(Database issue):D296-9. 2004 Jan I

II. Alm RA, Ling LS, Moir DT, King BL, Brown ED, Doig PC, Smith DR, Noonan B, Guild BC, dejonge BL, Carmel G, Tummino PJ, Caruso A, Uria-Nickelsen M, Mills DM, Ives C, Gibson R, Merberg D, Mills SD, Jiang Q, Taylor DE, Vovis GF, Trust T]: Genomic-sequence comparison of two unrelated isolates of the human gastric pathogen Helicobacter pylori. Nature 1999, 397(67 I 5): I 76-80.

12. Hayashi T, Makino K, Ohnishi M, Kurokawa K, Ishii K, Yokoyama K, Han CG, Ohtsubo E, Nakayama K, Murata T, Tanaka M, Tobe T, lida T, Takami H, Honda T, Sasakawa C, Ogasawara N, Yasunaga T, Kuhara S, Shiba T, Hattori M, Shinagawa H: Complete genome sequence of enterohemorrhagic Escherichia coli O157:H7 and genomic comparison with a laboratory strain K-I2. DNA Res 2001, 8(I): I |-22.

13. Perna NT, Plunkett G 3rd, Burland V, Mau B, Glasner JD, Rose DJ, Mayhew GF, Evans PS, Gregor J, Kirkpatrick HA, Posfai G, Hackett J, Klink S, Boutin A, Shao Y, Miller L, Grotbeck EJ, Davis NW, Lim A, Dimalanta ET, Potamousis KD, Apodaca J, Anantharaman TS, Lin J, Yen G, Schwartz DC, Welch RA, Blattner FR: Genome sequence of enterohaemorrhagic Escherichia coli O I 57:H7. Nature 200I, 409(68 I 9):529-33.

14. Canchaya C, Fournous $\mathrm{G}$, Brussow $\mathrm{H}$ : The impact of prophages on bacterial chromosomes. Mol Microbiol 2004, 53(I):9-I8.

15. Schneider D, Duperchy E, Depeyrot J, Coursange E, Lenski R, Blot M: Genomic comparisons among Escherichia coli strains B, K-I 2, and OI57:H7 using IS elements as molecular markers. BMC Microbiol 2002, 2(I): I8.

16. E. coli O I57:H7 Sakai Genome Information, KI 2 and Sakai loop coordinates 2002 [http://genome.gen-info.osaka-u.ac.jp/cgibin/ol 57/loop.pl?table=loop].

17. Reid SD, Herbelin CJ, Bumbaugh AC, Selander RK, Whittam TS: Parallel evolution of virulence in pathogenic Escherichia coli. Nature 2000, 406(679I):64-7.
18. Konstantinidis KT, Tiedje JM: Genomic insights that advance the species definition for prokaryotes. Proc Natl Acad Sci U S A 2005, I02(7):2567-72. 2005 Feb I5

19. Hoebeke M, Nicolas P, Bessieres P: MuGeN: simultaneous exploration of multiple genomes and computer analysis results. Bioinformatics 2003, I9(7):859-64.

20. Blattner FR, Plunkett G 3rd, Bloch CA, Perna NT, Burland V, Riley M, Collado-Vides J, Glasner JD, Rode CK, Mayhew GF, Gregor J, Davis NW, Kirkpatrick HA, Goeden MA, Rose DJ, Mau B, Shao Y: The complete genome sequence of Escherichia coli K-I2. Science 1997, 277(533I): |453-74.

21. Welch RA, Burland V, Plunkett G 3rd, Redford P, Roesch P, Rasko D, Buckles EL, Liou SR, Boutin A, Hackett J, Stroud D, Mayhew GF, Rose DJ, Zhou S, Schwartz DC, Perna NT, Mobley HL, Donnenberg MS, Blattner FR: Extensive mosaic structure revealed by the complete genome sequence of uropathogenic Escherichia coli. Proc Natl Acad Sci USA 2002, 99(26): I 7020-4.

22. Bachellier S, Clement JM, Hofnung $M$ : Short palindromic repetitive DNA elements in enterobacteria: a survey. Res Microbiol 1999, I50(9-10):627-639.

23. E. coli KI2 BIMES 1999 [http://www.pasteur.fr/recherche/unites/ pmtg/repet/tableauBIMEcoli.html].

24. Bachellier S, Clement JM, Hofnung M, Gilson E: Bacterial interspersed mosaic elements (BIMEs) are a major source of sequence polymorphism in Escherichia coli intergenic regions including specific associations with a new insertion sequence. Genetics 1997, I 45(3):551-62.

25. Brussow H, Canchaya C, Hardt WD: Phages and the evolution of bacterial pathogens: from genomic rearrangements to lysogenic conversion. Microbiol Mol Biol Rev 2004, 68(3):560-602.

26. Viguera $E$, Canceill D, Ehrlich SD: Replication slippage involves DNA polymerase pausing and dissociation. EMBO J 200I, 20(10):2587-95.

27. Tamas I, Klasson L, Canback B, Naslund AK, Eriksson AS, Wernegreen JJ, Sandstrom JP, Moran NA, Andersson SG: 50 million years of genomic stasis in endosymbiotic bacteria. Science 2002, 296(5577):2376-9.

28. Wei J, Goldberg MB, Burland V, Venkatesan MM, Deng W, Fournier G, Mayhew GF, Plunkett G 3rd, Rose DJ, Darling A, Mau B, Perna NT, Payne SM, Runyen-Janecky LJ, Zhou S, Schwartz DC, Blattner FR: Complete genome sequence and comparative genomics of Shigella flexneri serotype 2 a strain 2457T. Infect Immun 2003, 7 I (5):2775-86

29. Darling AC, Mau B, Blattner FR, Perna NT: Mauve: multiple alignment of conserved genomic sequence with rearrangements. Genome Res 2004, I 5(I): I84-194.

30. Glasner JD, Liss P, Plunkett G 3rd, Darling A, Prasad T, Rusch M, Byrnes A, Gilson M, Biehl B, Blattner FR, Perna NT: ASAP, a systematic annotation package for community analysis of genomes. Nucleic Acids Res 2003, 3 I ( I ): |47-5 I | 4. I394-403

31. Ohnishi M, Terajima J, Kurokawa K, Nakayama K, Murata T, Tamura K, Ogura Y, Watanabe H, Hayashi T: Genomic diversity of enterohemorrhagic Escherichia coli OI57 revealed by whole genome PCR scanning. Proc Natl Acad Sci U S A 2002 , 99(26): I 7043-8. 2002 Dec 24; Epub 2002 Dec 12

32. Emboss [http://emboss.sourceforge.net/]

Publish with Bio Med Central and every scientist can read your work free of charge

"BioMed Central will be the most significant development for disseminating the results of biomedical research in our lifetime. "

Sir Paul Nurse, Cancer Research UK

Your research papers will be:

- available free of charge to the entire biomedical community

- peer reviewed and published immediately upon acceptance

- cited in PubMed and archived on PubMed Central

- yours - you keep the copyright 